\title{
Videojuegos educativos enfocados a la enseñanza de ciberseguridad para estudiantes escolares
}

\author{
Miguel Antonio Plácido-Morales
}

La navegación en internet se ha tornado más insegura para todos con el paso de los años, pero distintas investigaciones en el mundo y en Latinoamérica han evidenciado que dentro de este panorama los jóvenes son objetivos más vulnerables para los atacantes en la red. En nuestro país esto es una realidad que puede ser combatida con el uso de métodos educativos modernos, como los videojuegos.

\section{Educational Video Games Focused on Teaching Cybersecurity to School Students}

Internet browsing has increasingly become unsafe for everyone over the years. In this context, different research projects conducted around the world and in Latin America have shown that young people are the most vulnerable targets for network attackers. In our country, this is a real situation that can be fought by using modern educational methods such as video games. 


\title{
Videojuegos educativos enfocados a la enseñanza de ciberseguridad para estudiantes escolares
}

\author{
Miguel Antonio Plácido-Morales \\ 20141039@aloe.ulima.edu.pe
}

\section{Resumen}

La navegación en internet se ha tornado más insegura para todos con el paso de los años, pero distintas investigaciones en el mundo y en Latinoamérica han evidenciado que dentro de este panorama los jóvenes son objetivos más vulnerables para los atacantes en la red. En nuestro país esto es una realidad que puede ser combatida con el uso de métodos educativos modernos, como los videojuegos.

\section{Introducción}

En el Perú se estima que casi el $44 \%$ de jóvenes actualmente conectados han sido acosados alguna vez en internet y muchos de estos casos no son denunciados formalmente. La enseñanza de seguridad en la red actualmente no está contemplada en el currículo escolar nacional vigente.

Para combatir este problema se han empleado distintos métodos de enseñanza modernos para brindar a los jóvenes mayor conocimiento acerca de su seguridad personal en la red. Uno de estos métodos es el uso de "Serious Games", que permite a través de videojuegos enseñar a los alumnos contenido educativo.

Metodología
Para aumentar el conocimiento general sobre la cibersegurid
y la navegación segura en línea de los alumnos se utilizó
marco referencial K12 (específicamente el concep
"Descripción general de redes e Internet") como guía
desarrollo.
Los 3 subtemas principales tratados fueron:
- Subtema 1: educar sobre el uso de protección en líne
- Subtema 2: reconocer los tipos de informaciones
línea y comportamientos durante la navegación
- Subtema 3: enseñar los peligros de los ataques
seguridad en la red

Para el desarrollo del juego se empleó Unity3d junto con assets de libre uso para traer una experiencia de juego con gráficos modernos y con una historia que englobe el contenido educativo sobre la seguridad en la red.

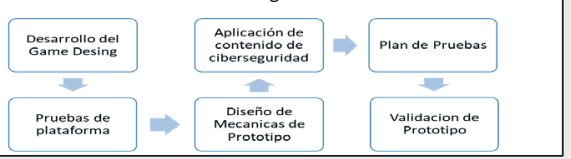

○

○
○

$\bigcirc$

\section{Resultados}

Se evaluará a 2 grupos de alumnos con diferentes cantidades, el primero para probar la efectividad del prototipo y el segundo para evaluar resultados con un incremento en la muestra.

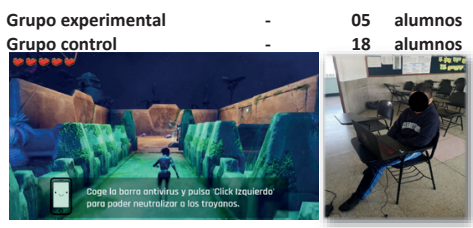

Se les evaluó a través de 2 pruebas: prueba de entrada y prueba de salida, para poder cuantificar de manera estadística el aprendizaje de los alumnos después de emplear el prototipo.

En los siguientes resultados se muestra el aumento de las respuestas correctas en cada subtema en porcentajes después de que los alumnos emplearan el prototipo en ambos grupos.

\begin{tabular}{|c|c|c|c|}
\hline & Subtema & Subtema & Subtema \\
\hline Grupo experimental & $\mathbf{1}$ & $\mathbf{2}$ & $\mathbf{3}$ \\
\hline Grupo control & $37 \%$ & $60 \%$ & $70 \%$ \\
\hline
\end{tabular}

\section{Conclusiones}

El videojuego educativo fue evaluado con éxito con 23 alumnos de un grado promedio a tercero de secundaria, además, se obtuvo una mejora en el aprendizaje de ciberseguridad y técnicas de navegación en ambos grupos.

Por otro lado, observamos que al ampliar la muestra esta afecta los resultados, siendo más precisos los que poseen una mayor cantidad de alumnos, lo que puede variar en un mínimo de $10 \%$ y un máximo de aproximadamente $40 \%$ de decremento por subtema según lo que analizamos en los resultados del grupo de control frente al grupo experimental.

\begin{tabular}{lc}
\hline Referencias & \\
Silva, C. S., Barbosa, G. A. R., Silva, I. S., & Ministerio de Educación del Perú. (2016). \\
Silva, T. S., Mourão, F., y Coutinho, F. & Currículo Nacional de la Educación Básica. \\
(2017, July 10). Privacy for Children and & 224. \\
Teenagers on Social Networks from a & Kaspersky. (2018). Kaspersky Lab registra un \\
Usability Perspective. & alza de 60 \% en ataques cibernéticos en \\
K-12 Computer Science Framework. (2016). & América Latina. \\
http://www.k12cs.org. & Pardo Angulo, F. . . (2018). Los videojuegos \\
Jin, G., Tu, M., Kim, T.-H., Heffron, J., y & como herramienta pedagógica para \\
White, J. (2018, February 21). Game based & contribuir en el fortalecimiento de la \\
Cybersecurity Training for High School & competencia resolución de problemas. \\
Students. 68-73. &
\end{tabular}

$\bigcirc$

○

○ 